\title{
Augmenting Second Harmonic Generation Using Fano Resonances in Plasmonic Systems
}

\author{
Krishnan Thyagarajan, Jérémy Butet, and Olivier J. F. Martin* \\ Nanophotonics and Metrology Laboratory (NAM), Swiss Federal Institute of Technology Lausanne (EPFL), 1015, Lausanne, \\ Switzerland
}

\section{Supporting Information}

\begin{abstract}
Significant augmentation of second harmonic generation using Fano resonances in plasmonic heptamers made of silver is theoretically and experimentally demonstrated. The geometry is engineered to simultaneously produce a Fano resonance at the fundamental wavelength, resulting in a strong localization of the fundamental field close to the system, and a higher order scattering peak at the second harmonic wavelength. These results illustrate the versatility of Fano resonant structures to engineer specific optical responses both in the linear and nonlinear regimes thus paving the way for future investigations on the role of dark modes in nonlinear and quantum optics.
\end{abstract}
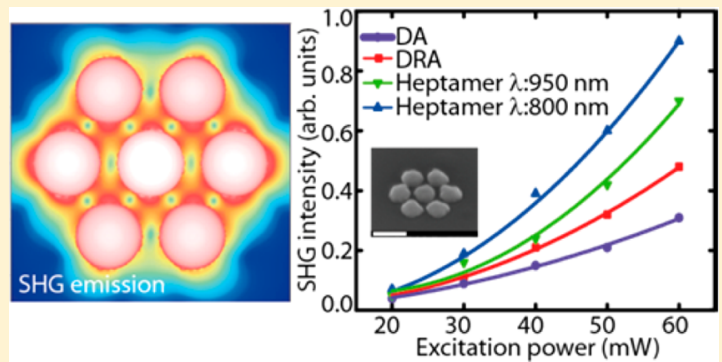

KEYWORDS: Plasmonics, nonlinear optics, Fano resonance, silver nanostructures, heptamers

$T^{1}$ he collective oscillations of electrons in the form of plasmon resonances in metallic nanostructures exhibit unique optical properties. ${ }^{1}$ One such property is the generation of hot spots, whose presence in nanostructures, such as optical antennae, is promising for applications in nonlinear optics, since they require a strong electric near-field. ${ }^{2}$ Recent studies have reported the observation of different nonlinear optical phenomena in such nanosystems, like, for example, second harmonic generation (SHG), ${ }^{3}$ multiphoton luminescence, ${ }^{4,5}$ third harmonic generation, ${ }^{6,7}$ higher harmonic generation, ${ }^{8}$ and four-wave mixing. ${ }^{9}$ SHG is significantly dependent on the symmetry of both the material being used and the structure being studied and therefore is a sensitive tool for characterizing plasmonic structures. ${ }^{10-13}$ SHG from centrosymmetric materials is due to the breaking of inversion symmetry at their surfaces and is therefore used as a surface probe. ${ }^{14}$ In recent years, strong SHG has been observed in metallic nanostructures such as sharp metallic tips, ${ }^{15}$ multiple resonant nanostructures, ${ }^{16,17}$ split-ring resonators, ${ }^{18}$ metamaterials ${ }^{19,20}$ and nanocups, ${ }^{21}$ underlining the fast growing interest in the area of nonlinear plasmonics. ${ }^{22,23}$ Efficient SHG requires the presence of strong SHG sources, that is, nonlinear polarization currents oscillating at the second harmonic frequency, at the nanostructure surface as well as an efficient scattering of the SHG signal into the far-field. Several strategies have been developed to enhance SHG from metallic nanostructures including using multiple resonances at both the fundamental and the second harmonic wavelengths, ${ }^{16}$ breaking the centrosymmetry using noncentrosymmetric nanostructures ${ }^{20}$ and even enhancing the electric fields using nanogaps. $^{24}$ However, the efficiency of SHG is restricted by two fundamental classes of losses that electromagnetic waves suffer in such metallic structures, namely radiative "losses" like optical scattering and nonradiative losses like the generation of heat. ${ }^{25}$ Therefore, to increase the detected SHG in the far-field the structures must decrease the radiative losses at the fundamental wavelength while increasing them at the second harmonic wavelength and at the same time increase the near-field at the fundamental wavelength. Because the SHG yield increases as the square of the fundamental field intensity, ${ }^{26}$ a higher near-field at the fundamental is required to increase the near-field at the second harmonic. The width of a plasmonic resonance indicates the total losses, both radiative and nonradiative, that the structure suffers. A narrower plasmonic resonance therefore implies lower total losses and prior work has been done to tune the plasmon resonance width by modifying the structures. ${ }^{27,28}$ However, due to the lack of complete control over the width, this is not a very effective means to control the generated local near-field.

Fano resonances, on the other hand, allow for an easy manipulation of the plasmon resonance width and the near-field. The most common line shape of a resonance is a Lorentzian and typically plasmon resonances are Lorentzian in shape. Fano resonances, on the other hand, are asymmetric in their line shape. ${ }^{29-32}$ Such resonances first arose in the work of Ugo Fano in atom spectroscopy where the characteristic asymmetric line shape is produced by the interference between a discrete state and a continuum. ${ }^{33}$ In plasmonics, Fano resonances are produced by the interaction between modes of the system to result in a subradiant (dark) mode and a superradiant (bright) mode. ${ }^{34} \mathrm{~A}$ bright mode is one which possesses a large dipole moment and can therefore be excited under plane wave illumination as well as scatter into the farfield. On the other hand, a dark mode having a lower dipole moment cannot interact directly with the plane wave or be scattered into the far-field. However, it can be excited by the near-field associated with the bright mode. Therefore, the plane wave can first

Received: February 20, 2013

Revised: $\quad$ March 22, 2013

Published: March 27, 2013 

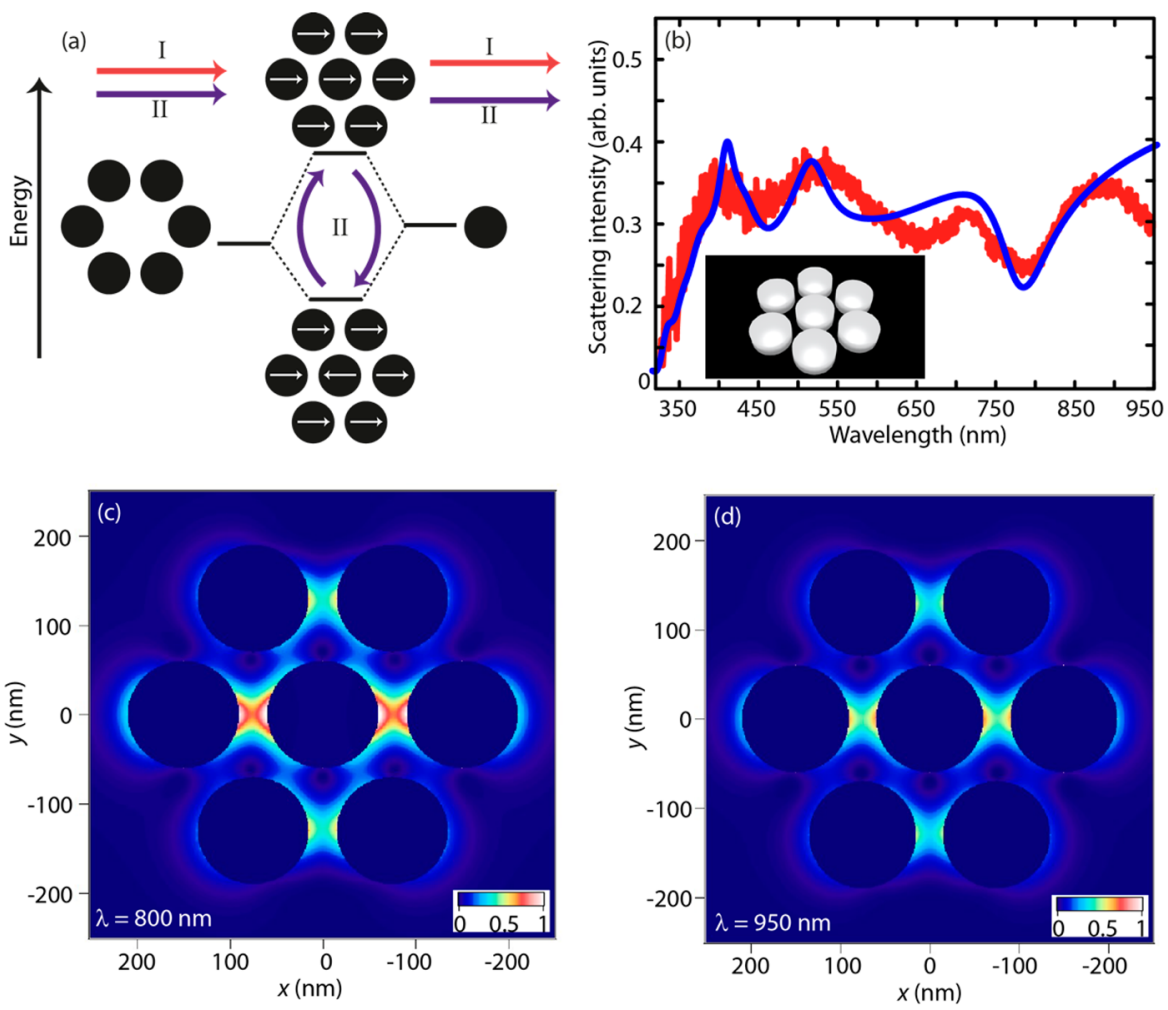

Figure 1. (a) Schematic representation of the plasmon hybridization in a heptamer and the associated interaction between the bright and dark modes through the interference of paths I and II, leading to the Fano resonance, (b) SIE computed (blue) and experimentally measured (red) optical scattering cross section for the heptamers, note the scattering dip at $\lambda=800 \mathrm{~nm}$ and the peak at $\lambda=400 \mathrm{~nm}$. Near-field intensity enhancement distribution of the fundamental intensity shown on a normalized linear scale for (c) $\lambda=800 \mathrm{~nm}$ and (d) $\lambda=950 \mathrm{~nm}$ incidence wavelength, close to an idealized silver heptamer. The structures have a $120 \mathrm{~nm}$ diameter, $30 \mathrm{~nm}$ height, and $30 \mathrm{~nm}$ gap.

excite the bright mode, which then excites the dark mode, which in turn interferes with the scattering from the same bright mode. Depending on the phase difference between both pathways, the final response of the bright mode can be either attenuated or reinforced. The frequency dependence of the phase and therefore the position of the attenuated or reinforced features of the Fano resonance, allows us to engineer the optical properties of the nanosystem.

In this Letter, we compare the SHG from plasmonic systems that exhibit a Fano resonance and those that do not, demonstrating the implication of dark mode and enhanced near-fields to produce strong far-field SHG. For this work, we have chosen the plasmonic heptamer as the Fano resonant system because the observation of Fano resonances in such a nanosystem does not require any symmetry breaking for inducing the coupling between the dark mode and the bright mode. ${ }^{35-37}$ Indeed, SHG is known to be very sensitive to the symmetry and symmetry breaking will induce further effects impacting the SHG. To the best of our knowledge, this is the first time that a Fano resonance is used for increasing SHG from plasmonic nanostructures. We engineer the silver heptamers to obtain the Fano dip at the fundamental wavelength $\lambda=800 \mathrm{~nm}$ and a higher order scattering peak at the second harmonic wavelength $\lambda$ $=400 \mathrm{~nm}$. To qualitatively visualize its response, one can imagine the heptamer as a simpler subsystem consisting of an outer ring hexamer and an individual central unit, which hybridize to create two modes, the optical bright mode and the optical dark mode as can be seen in Figure 1a. It is these two modes that interact to produce the Fano resonance. ${ }^{36,37}$ By having a minimum in the optical scattering cross-section and therefore a destructive interference of the scattering in the far-field, at the excitation wavelength, we ensure that rather than being scattered away into the far-field, the fundamental laser field remains strong in the nearfield, thereby providing a greater conversion into the second harmonic signal. The corresponding dimensions of the optimized heptamer are a $120 \mathrm{~nm}$ diameter, $30 \mathrm{~nm}$ height, and $30 \mathrm{~nm}$ gap between the individual units. By choosing silver as the working metal we are able to obtain the attenuated dip at $\lambda=800 \mathrm{~nm}$, which is the fundamental wavelength of the Ti:Sapphire laser source that we use for our nonlinear optical experiments. The optical properties of the heptamer have been computed using the surface integral equation (SIE) formulation. ${ }^{38}$ Note that silver is a preferred material for these structures due to its particular wavelength tunability close to the Fano profile. ${ }^{39}$ Figure $1 b$ shows the scattering cross-section as a function of the wavelength calculated for such a heptamer. The optical response clearly fulfills the criteria discussed above. In order to verify that these lead to SHG enhancement, further computations have been performed. Figure $1 c, d$ shows the nearfield intensity computed for an incident wavelength of $\lambda=800 \mathrm{~nm}$ and $\lambda=950 \mathrm{~nm}$, respectively, polarized along the $x$-direction. The linear plots in Figure 1c,d are normalized to 1 . As expected, the near-field intensity is $30 \%$ stronger when the incident wavelength is tuned close to the scattering Fano dip than when not tuned at the dip. The second harmonic electric near-field is computed for two different fundamental wavelengths using the method developed by Mäkitalo et al. ${ }^{40}$ Note that only the component $\chi_{\text {surf,nnn }}$ of the surface tensor, where $n$ denotes the component normal to the surface, is considered. Indeed, this component is known to be the main surface contribution to SHG from metallic nanoparticles. ${ }^{41,42}$ Recent experimental work on the SHG from spherical silver 

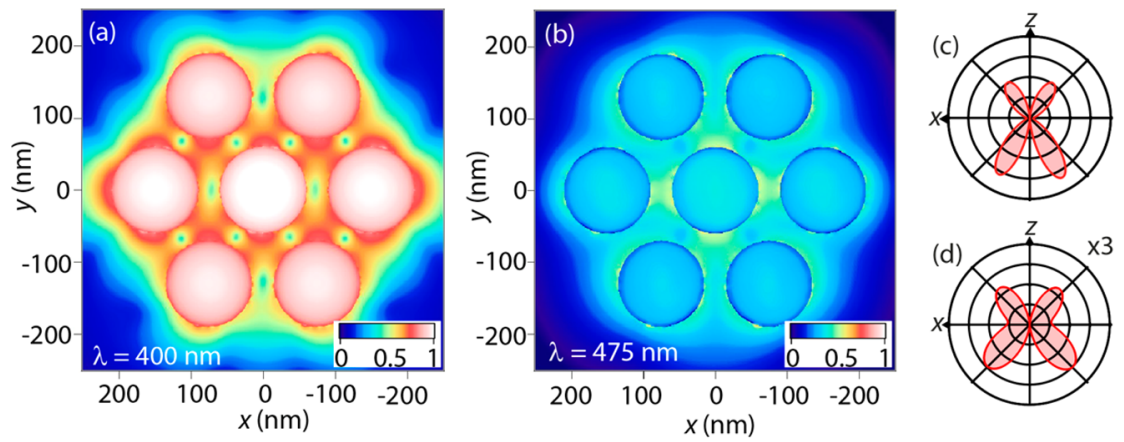

Figure 2. Near-field distribution of the second harmonic intensity close to an ideal silver heptamer plotted on a logarithmic scale with SHG at (a) $\lambda$ $=400 \mathrm{~nm}$ (corresponding to the fundamental at $\lambda=800 \mathrm{~nm}$ in Figure 1c) and (b) $475 \mathrm{~nm}$ (corresponding to the fundamental at $\lambda=900 \mathrm{~nm}$ in Figure 1d), the angular intensity emission plots for the two corresponding cases of (c) SHG at $\lambda=400 \mathrm{~nm}$ and (d) SHG at $\lambda=475 \mathrm{~nm}$. Note that in panel (d), the intensity has been scaled by a factor of 3 showing the much stronger SHG in the far-field for the Fano-resonant case.

nanoparticles has further demonstrated that the nnn component represents the dominant contribution to the second harmonic signal and other contributions play only a minor role in the SHG from plasmonic nanostructures. ${ }^{43}$ Figure 2 shows the second harmonic near-field intensity when the fundamental wavelength is (a) $\lambda=800 \mathrm{~nm}$ and (b) $\lambda=950 \mathrm{~nm}$. The logarithmic plots in Figure $2 \mathrm{a}, \mathrm{b}$ are normalized with 1 representing the maximum second harmonic intensity in both cases. A drastic enhancement of the near-field at the second harmonic wavelength in the Fanoresonant system is clearly visible: in that case, the near-field intensity is about 25 times stronger $(\lambda=400$, Figure $2 \mathrm{a})$ than in the off-Fano resonant case $(\lambda=475$, Figure $2 b)$. To further enhance the SHG in the far-field, the structure was engineered to exhibit strong scattering at the second harmonic wavelength (see the scattering spectrum Figure.1b). This is visible in Figure $2 \mathrm{c}, \mathrm{d}$ where we show the angular distribution for second harmonic at $\lambda=400 \mathrm{~nm}$ and $\lambda=$ $475 \mathrm{~nm}$, respectively. The emission patterns observed do not correspond to dipolar emission but to higher multipolar emission. ${ }^{44}$ The determination of the multipolar modes involved is not straightforward and is beyond the scope of the present letter. Note that the SHG vanishes in the forward and backward directions as expected for a centrosymmetric nanostructure. While the angular distribution is similar for both excitation wavelengths, the intensity of the SHG in the off-Fano resonant case corresponds to $35 \%$ of the second harmonic intensity in the on-Fano resonant case (note that the data are scaled by $3 x$ in Figure $2 d$ ).

To experimentally verify this, the silver heptamers were fabricated using electron beam lithography followed by metal evaporation. Silver is a very difficult metal to work with because of its ease of oxidation, which prevents the realization of ideal structures with small dimensions and gaps due to the large grain size. Through a process of optimization, we were able to reproducibly fabricate silver heptamers on a fused silica substrate with gaps down to $20 \mathrm{~nm}$, as can be seen in Figure 3 . Nonlinear optical experiments were conducted on an array of such silver heptamers as is visible in Figure 3a. A close-up of one such silver heptamer structure is visible in Figure $3 \mathrm{~b}$.

Linear optical measurements were carried out on the silver heptamers using a dark-field microscope (Olympus IX71 with a $60 \times 0.7$ NA objective). The measured linear optical scattering spectrum shown in Figure $1 \mathrm{~b}$ clearly indicates that the fabricated heptamers exhibit the Fano dip at the desired wavelength of about $\lambda=800 \mathrm{~nm}$. The asymmetric nature of the Fano resonant system is clearly visible. What can be further concluded is that using a laser at $\lambda=800 \mathrm{~nm}$ ensures a low optical scattering, while a laser at $\lambda=950 \mathrm{~nm}$ shifts the optical scattering to a larger value away from
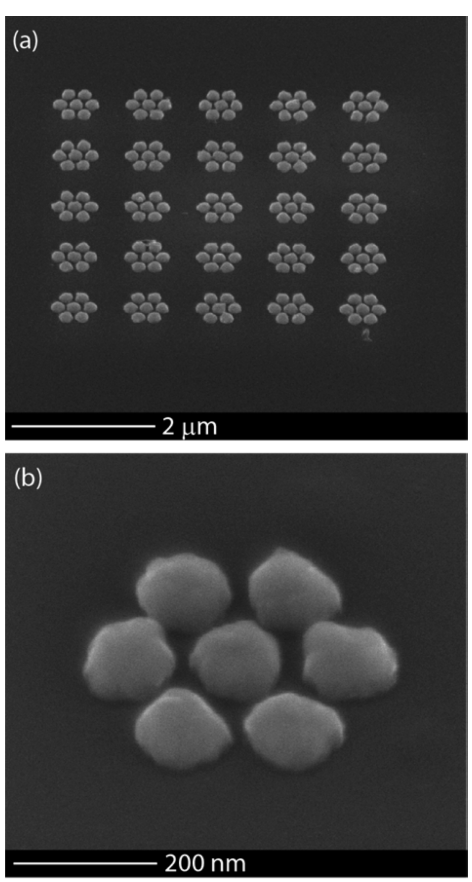

Figure 3. Scanning electron microscopy images of the fabricated silver heptamers showing (a) an array of structures and (b) a close-up of a single heptamer. The dimensions are a $120 \mathrm{~nm}$ diameter, a $30 \mathrm{~nm}$ height, and a $30 \mathrm{~nm}$ gap between adjacent structures.

the dip. To conduct the nonlinear optical experiments, a tunable Ti:Sapphire laser (Coherent Chameleon femtosecond laser: $150 \mathrm{fs}$, $80 \mathrm{MHz}$ ) connected to a Leica MP5 multiphoton microscope with a Leica HCX APO 20× NA 1 water immersion objective was used. The laser beam was made incident on the sample and the SHG measured in transmission. Choosing the lasing wavelength to be either $\lambda=800 \mathrm{~nm}$ or $\lambda=950 \mathrm{~nm}$, we were able to tune the excitation wavelength either at the Fano dip or off the Fano dip. To compare Fano-resonant structures with more conventional plasmonic nanostructures, SHG measurements were performed for three types of plasmonic nanostructures: the heptamer (both on and off-Fano resonance), a dipole antenna (DA), and a double resonance antenna (DRA). ${ }^{16}$ The DA and the DRA are made of aluminum. The DA, DRA, and on-Fano resonance heptamer were excited at $\lambda=800 \mathrm{~nm}$, while the off-Fano resonance heptamer was excited at $\lambda=950 \mathrm{~nm}$. The SHG measurements on the three different structures are reported in Figure 4. The DA is one of the more basic plasmonic structures studied for SHG; it can resonate 


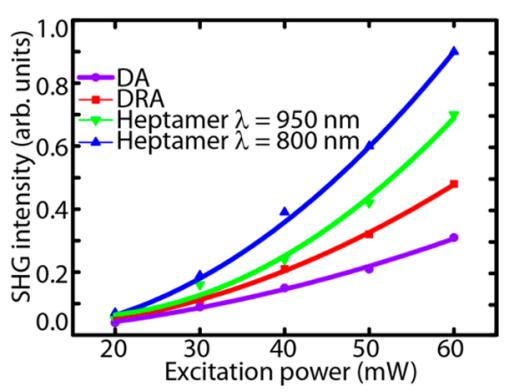

Figure 4. Nonlinear optical experimental results comparing the SHG in the far-field for the silver heptamer excited at the Fano resonance $(\lambda=800 \mathrm{~nm})$ and off-Fano resonance $(\lambda=950 \mathrm{~nm})$, as well as for a dipole antenna (DA) and a double resonant antenna (DRA). Clearly, the SHG under the Fano resonance excitation is 3 times stronger than the DA. The lines are quadratic fits to the experimental points.

only at one wavelength of interest, either the fundamental or the second harmonic. The DRA based on double resonances both at the fundamental and second harmonic produces a 1.5 stronger SHG intensity than the DA (see Figure 4). ${ }^{16}$ This finite improvement is due to the additional resonance at the second harmonic wavelength. The silver heptamer has more gaps where the field is enhanced and therefore even off-Fano resonance, exhibits a 2.7 times stronger SHG as compared to the DA. Under on-Fano resonance excitation, the SHG from the heptamer is enhanced 3 times when compared to the standard DA. It is clearly visible from Figure 4 that the SHG in the far-field is the greatest for the silver heptamer excited with a laser wavelength that matches the Fano dip. Such a significant SHG enhancement, especially when compared to the DA, indicates the major role of the near-field enhancement and how this phenomenon can be tuned and reinforced in a Fano system. Nevertheless, a lower augmentation of the experimentally SHG is observed as compared to the theoretical predictions. This can be explained by fabrication imperfections in the array as discussed in the Supporting Information (S1). Fabrication variations in individual structures can indeed play a nontrivial role in the SHG from plasmonic nanostructures. ${ }^{13,45}$

In summary, the SHG from a Fano resonant system has been studied both theoretically and experimentally. The nonlinear SHG in the engineered silver heptamers is shown to be significantly stronger when utilizing the generation of a high near-field at the fundamental wavelength by reducing scattering losses, while assuring a strong scattering at the second harmonic. These results illustrate the versatility of Fano resonant structures to engineer specific optical responses both in the linear and nonlinear regimes thus paving the way for future investigations on the role of dark modes in nonlinear optics ${ }^{46}$ as well as possible applications in quantum optics. $^{47}$

\section{ASSOCIATED CONTENT}

\section{S Supporting Information}

Additional information and figures. This material is available free of charge via the Internet at http://pubs.acs.org.

\section{AUTHOR INFORMATION}

\section{Corresponding Author}

*E-mail: olivier.martin@epfl.ch.

\section{Notes}

The authors declare no competing financial interest.

\section{ACKNOWLEDGMENTS}

The authors would like to acknowledge C. Santschi for help in optimizing the fabrication process of the structures, T. Laroche from the BioImaging and Optics Platform (BiOP) where part of the work was performed, B. Gallinet for stimulating discussions, and funding from the Swiss National Science Foundation (Grant 200021_132694).

\section{REFERENCES}

(1) Maier, S. A. Plasmonics: Fundamentals and Applications; Springer: New York, 2007.

(2) Slablab, A.; Xuan, L.; Zielinski, M.; de Wilde, Y.; Jacques, V.; Chauvat, D.; Roch, J.-F. Opt. Express 2012, 20, 220-227.

(3) Hanke, T.; Krauss, G.; Träutlein, D.; Wild, B.; Bratschitsch, R.; Leitenstorfer, A. Phys. Rev. Lett. 2009, 103, 257404-4.

(4) Castro-Lopez, M.; Brinks, D.; Sapienza, R.; van Hulst, N. F. Nano Lett. 2011, 11, 4674-4678.

(5) Biagioni, P.; Brida, D.; Huang, J.-S.; Kern, J.; Duo, L.; Hecht, B.; Finazzi, M.; Cerullo, G. Nano Lett. 2012, 12, 2941-2947.

(6) Hentschel, M.; Utikal, T.; Giessen, H.; Lippitz, M. Nano Lett. 2012, 12, 3778-3782.

(7) Navarro-Cia, M.; Maier, S. A. ACS Nano 2012, 6, 3537-3544.

(8) Kim, S.; Jin, J.; Kim, Y.-J.; Park, I.-Y.; Kim, Y.; Kim, S.-W. Nature 2008, 453, 757-760.

(9) Danckwerts, M.; Novotny, L. Phys. Rev. Lett. 2007, 98, 026104-4.

(10) Butet, J.; Duboisset, J.; Bachelier, G.; Russier-Antoine, I.; Benichou, E.; Jonin, C.; Brevet, P.-F. Nano Lett. 2010, 10, 1717-1721.

(11) Valev, V. K.; Silhanek, A. V.; Verellen, N.; Gillijns, W.; Van Dorpe, P.; Aktsipetrov, O. A.; Vandenbosch, G. A. E.; Moshchalkov, V. V.; Verbiest, T. Phys. Rev. Lett. 2010, 104, 127401-4.

(12) Husu, H.; Siikanen, R.; Mäkitalo, J.; Lehtolahti, J.; Laukkanen, J.; Kuittinen, M.; Kauranen, M. Nano Lett. 2012, 12, 673-677.

(13) Butet, J.; Thyagarajan, K.; Martin, O. J. F. Nano Lett. 2013, DOI: $10.1021 / \mathrm{nl} 400393 \mathrm{e}$.

(14) Roke, S.; Gonella, G. Annu. Rev. Phys. Chem. 2012, 63, 353-378.

(15) Neacsu, C. C.; Reider, G. A.; Raschke, M. B. Phys. Rev. B 2005, $71,201402-4$.

(16) Thyagarajan, K.; Rivier, S.; Lovera, A.; Martin, O. J. F. Opt. Express 2012, 20, 12860-12865.

(17) Harutyunyan, H.; Volpe, G.; Quidant, R.; Novotny, L. Phys. Rev. Lett. 2012, 108, 217403-4.

(18) Cirac, C.; Poutrina, E.; Scalora, M.; Smith, D. R. Phys. Rev. B 2012, 86, 115451-10.

(19) Wurtz, G. A.; Pollard, R.; Hendren, W.; Wiederrecht, G. P.; Gosztola, D. J.; Podolskiy, V. A.; Zayats, A. V. Nat. Nanotechnol. 2011, $6,107-111$.

(20) Husu, H.; Siikanen, R.; Mäkitalo, J.; Lehtolahti, J.; Laukkanen, J.; Kuittinen, M.; Kauranen, M. Nano Lett. 2012, 12, 673-677.

(21) Zhang, Y.; Grady, N. K.; Ciceron, A. -O.; Halas, N. J. Nano Lett. 2011, 11, 5519-5523.

(22) Noskov, R.; Belov, P.; Kivshar, Y. Sci. Rep. 2012, 2, 873-8.

(23) Kauranen, M.; Zayats, A. V. Nat. Photonics 2012, 6, 737-748.

(24) Palomba, S.; Danckwets, M.; Novotny, L. J. Opt. A: Pure Appl. Opt. 2009, 11, 114030-6.

(25) Chang, W.-S.; Willingham, B.; Slaughter, L. S.; DominguezMedina, S.; Swanglap, P.; Link, S. Acc. Chem. Res. 2012, 45, 19361945.

(26) Boyd, R. W. Nonlinear optics; Academic Press: New York, 1992.

(27) Sonnichsen, C.; Franzl, T.; Wilk, T.; von Plessen, G.; Feldmann, J. Phys. Rev. Lett. 2002, 88, 077402-4.

(28) Hanke, T.; Cesar, J.; Knittel, V.; Trugler, A.; Hohenester, U.; Leitenstorfer, A.; Bratschitsch, R. Nano Lett. 2012, 12, 992-996.

(29) Luk'yanchuk, B.; Zheludev, N. I.; Maier, S. A.; Halas, N. J.; Nordlander, P.; Giessen, H.; Chong, C. T. Nat. Mater. 2010, 9, 707715 .

(30) Miroshnichenko, A. E.; Flach, S.; Kivshar, Y. S. Rev. Mod. Phys. 2010, 82, 2257-2298. 
(31) Miroshnichenko, A. E.; Kivshar, Y. S. Nano Lett. 2012, 12, 6459-6463.

(32) Gallinet, B.; Martin, O. J. F. ACS Nano 2011, 5, 8999-9008.

(33) Fano, U. Phys. Rev. 1961, 124, 1866-1878.

(34) Gallinet, B.; Martin, O. J. F. Opt. Express 2011, 19, 2216722175.

(35) Hentschel, M.; Saliba, M.; Vogelgesang, R.; Giessen, H.; Alivisatos, P. A.; Liu, N. Nano Lett. 2010, 10, 2721-2726.

(36) Lassiter, J. B.; Sobhani, H.; Fan, J. A.; Kundu, J.; Capasso, F.; Nordlander, P.; Halas, N. J. Nano Lett. 2010, 10, 3184-3189.

(37) Hentschel, M.; Dregely, D.; Vogelgesang, R.; Giessen, H.; Liu, N. ACS Nano 2011, 5, 2042-50.

(38) Kern, A. M.; Martin, O. J. F. J. Opt. Soc. Am. A 2009, 26, 732740.

(39) Butet, J.; Bachelier, G.; Russier-Antoine, I.; Bertorelle, F.; Mosset, A.; Lascoux, N.; Jonin, C.; Benichou, E.; Brevet, P.-F. Phys. Rev. B 2012, 86, 075430-5.

(40) Mäkitalo, J.; Suuriniemi, S.; Kauranen, M. Opt. Express 2011, 23, 23386-23399.

(41) Wang, F. X.; Rodriguez, F. J.; Albers, W. M.; Ahorinta, J. E.; Sipe, J. E.; Kauranen, M. Phys. Rev. B 2009, 80, 233402-4.

(42) Bachelier, G.; Butet, J.; Russier-Antoine, I.; Jonin, C.; Benichou, E.; Brevet, P.-F. Phys. Rev. B 2010, 82, 235403-5.

(43) Gonella, G.; Gan, W.; Dai, H.-L. J. Phys. Chem. Lett. 2012, 3, 2877-2881.

(44) Butet, J.; Bachelier, G.; Russier-Antoine, I.; Jonin, C.; Benichou, E.; Brevet, P.-F. Phys. Rev. Lett. 2010, 105, 077401-4.

(45) Huttunen, M. J.; Bautista, G.; Decker, M.; Linden, S.; Wegener, M.; Kauranen, M. Opt. Mater. Express 2011, 1, 46-56.

(46) Chowdhury, D. R.; Singh, R.; Taylor, A. J.; Chen, H. -T.; Azad, A. K. Appl. Phys. Lett. 2013, 102, 011122-5.

(47) Dong, C.; Fiore, V.; Kuzyk, M. C.; Wang, H. Science 2012, 338, 1609-1613. 